\section{THROMBOTIC MICROANGIOPATHY}

BY

\author{
RUTH D. REVILL, M.D., M.R.C.P. \\ Formerly Resident Medical Officer, Gulson Hospital, \\ Coventry \\ AND
}

\section{W. C. MacC. WILSON, M.D., B.Sc, D.P.H. Senior Pathologist, Coventry Group Hospitals}

Thrombotic microangiopathic haemolytic anaemia (thrombotic microangiopathy) is the name proposed (Symmers, 1952) for a rarely recognized condition which presents the striking combination of acute haemolytic anaemia, thrombocytopenic purpura, fever, and fluctuating neurological disturbances. The aetiology is unknown. The macroscopic appearances at necropsy are neither specific nor particularly striking, but the microscopical finding of widespread patchy thrombosis affecting minute blood vessels is characteristic. There is no evidence of inflammation and little or none of tissue necrosis. The condition may be related to the so-called collagen diseases.

Symmers (1952) gives a comprehensive review of the literature and adds a full study of two personal cases. He could find only 31 undoubted cases recorded : most of these had been published in the United States, where the condition is usually known as thrombotic thrombocytopenic purpura, and only three from this country. $\mathrm{He}$ had himself jointly reported one of these under the title "platelet thrombosis syndrome" (Symmers and Barrowcliff, 1951).

The case recorded here confirms the findings of previous observers but adds no new information; the intention is to give only enough detail to justify acceptance of the case as genuine and to stimulate interest in the disease in the hope that earlier diagnosis and treatment may alter the present uniformly fatal prognosis and that further studies may elucidate the aetiology and doubtful points in the pathology.

\section{Case Report}

A man aged 38 was admitted to hospital with a history of having been unable to work for six months because of ulcers on the legs. These had improved recently under treatment with propamidine cream and zinc and castor oil ointment. The present illness began one week before admission and was of sudden onset with cramping abdominal pain and vomiting. The urine became red in colour at about the same time, and, as the condition was thought to be due to pyelonephritis, sulphadimidine, $2 \mathrm{~g}$. followed by $0.5 \mathrm{~g}$. six-hourly, was given. This was discontinued after two days, as the urine darkened in colour and haemorrhagic spots developed around the tongue and buccal mucous membrane. Icterus of the sclerae was first noted on the morning of admission to hospital, when he was seen by his doctor because of loss of consciousness when getting out of bed.

There were no significant previous illnesses and no familial illnesses of note. His occupation was described as a "benchworker" and did not involve exposure to any recognized hazard.

On admission he was noted to be a pallid, obese man who was gravely ill. His temperature was $99.6^{\circ} \mathrm{F}$. (37.6 C.), pulse 120 , and respirations 32 . There was considerable icterus of the sclerae and mucous membranes together with bleeding of the gums. He was drowsy and showed poor attention, but there were no abnormal neurological signs otherwise at that time. The fundi showed no abnormality.
A petechial rash was widely scattered but particularly plentiful in the axillae and groins. The Hess test was negative. There was marked scarring and brown pigmentation of the medial aspects of the lower limbs at the sites of the previous ulceration. Examination of the cardiovascular and respiratory systems revealed no abnormality other than the presence of crepitations at the left base. The blood pressure was $160 / 90$. The liver and spleen were not palpable and there was no adenopathy.

Laboratory Investigations.-Urine : Marked trace of albumin ; numerous red cells present ; no casts seen. Blood: $\mathrm{Hb}, 6.4 \mathrm{~g} . / 100 \mathrm{ml}$; red cells, $2,700,000$ per c.mm., white cells, 15,000 per c.mm. ; films showed a moderate polymorph leucocytosis with an occasional myelocyte; normoblasts were fairly numerous and red cells showed marked anisocytosis, poikilocytosis, polychromasia, and some anisochromia ; Group $\mathrm{O}$ rhesus-positive, no atypical antibodies ; Coombs test negative; platelets, approximately 90,000 ; bleeding-time, $6 \frac{1}{2}$ minutes; clotting-time, $11 \frac{1}{2}$ minutes (Wright's capillary method); serum bilirubin, $11.3 \mathrm{mg}$. per $100 \mathrm{ml}$.; colloidal gold, negative; thymol turbidity, 1.0 ; thymol flocculation, 0 ; alkaline phosphatase, 10 units; albumin, 4.2 g. per $100 \mathrm{ml}$; globulin, $3.3 \mathrm{~g}$. per $100 \mathrm{ml}$.; gamma globulin, $1 \mathrm{~g}$. per $100 \mathrm{ml}$.; Wassermann reaction and Kahn test had been found negative on a previous occasion.

Treatment was begun with penicillin, 500,000 units intramuscularly followed by 200,000 units at three-hourly intervals. As the patient's principal illness appeared to be that of an acute haemolytic anaemia complicated by purpura, A.C.T.H., $100 \mathrm{mg}$., and cortisone, $100 \mathrm{mg}$., were given by intramuscular injection some six hours after his admission to hospital. His general condition continued to worsen, and twelve hours after admission a right-sided hemiplegia developed. This was followed by coma and death shortly thereafter.

Summary of Necropsy Findings.-An extremely obese male. Moderate icterus. Widely scattered petechial rash with some haemorrhagic areas in an otherwise unexceptional eczematous rash on the lower limbs and groins. Bone marrow of sternum and ribs appeared active, but there was no replacement of fatty marrow in mid-shaft of right femur. The serous sacs contained small effusions of clear slightly icteric fluid. The heart showed extensive subepicardial petechiae and ecchymoses with some intramural haemorrhages. A few petechiae were also noted in kidneys, none elsewhere. Moderate splenomegaly (weight $410 \mathrm{~g}$.). Owing to an unfortunate misunderstanding the C.N.S. was not examined.

The microscopical findings were typical of thrombotic microangiopathy - that is, the partial or complete occlusion of numerous arterioles by hyaline or granular thrombus with a certain amount of endothelial reaction and absence of inflammatory cellular infiltration. The changes were maximal in the myocardium, but were also noted in the kidneys and spleen.

No vascular changes were apparent in the bone-marrow sections, which showed some degree of normoblastic hyperplasia and a considerable increase in megakaryocyte series, provisionally regarded as a reactive or compensatory phenomenon: the thrombocytopenia and occlusion of vessels are generally held to be due to formation of platelet thrombi, but this is not certain.

\section{Discussion}

The results of the laboratory investigations in life and the histological findings in the necropsy material conform exactly to those previously recorded, but shed no new light on the pathology of the condition.

The healed ulceration on the legs with eczematous rash may possibly have been a prodromal feature of the condition (urticarial rashes have been described), but its relevance or otherwise cannot be determined as the skin was not examined microscopically. 
An allergic or sensitization basis has been suggested for thrombotic microangiopathy, so the fact of local treatment with propamidine cream and zinc and castor oil ointment is recorded, although this may well be irrelevant.

The correct diagnosis had been suggested on the clinical features and the combination of an acute haemolytic anaemia with thrombocytopenic purpura. Owing to the patient's sudden death 12 hours after admission no information was gained concerning the value of treatment in this disease. Symmers (1952) gives a full discussion of various measures that have been tried. These include penicillin (no effect), sulphonamides (useless or possibly harmful), aureomycin (no effect), and streptomycin (doubtful, probably no effect). As there is no evidence that the condition is infective in origin further trial of this line of treatment is unlikely to be of value. Anticoagulants have not been tried owing to the marked haemorrhagic tendency already present. Blood transfusion had given either transient or no benefit owing to rapid haemolysis of the transfused cells. Platelet transfusion might lessen the thrombocytopenia, but there would appear to be a risk of increasing the thrombotic element. Replacement transfusion might be worth considering in conjunction with other measures, of which treatment with A.C.T.H. or cortisone seems to be the most promising. A.C.T.H. has been tried hitherto in only one case (Meacham et al., 1951), in which it may have been beneficial; the dosage given was small owing to the fear of ischaemic complications. Symmers points out that this objection may be unfounded. The place of splenectomy is doubtful, but it may be of value, perhaps as a long-term measure, if the patient could be tided over the most acute phase by other means.

\section{Summary}

A case of thrombotic microangiopathy is reported, only the sixth to be recorded in this country.

The salient features of the condition are fever, acute haemolytic anaemia, thrombocytopenia with purpura or other haemorrhagic manifestations, and fluctuating neurological signs.

The aetiology is unknown, but the morbid histology is characteristic-a widespread patchy thrombosis of minute blood vessels.

All cases so far recorded have been fatal, but earlier diagnosis would afford at least a hope of successful treatment; the condition may not be so rare as the extreme paucity of reports would suggest, and it should be considered in any case of febrile jaundice with haemorrhagic features.

Extensive reference has been made to a comprehensive paper on the subject which is readily accessible (Symmers, 1952).

We wish to thank Dr. J. C. Heather for permission to publish and Professor W. St. C. Symmers for confirming the histological diagnosis. Dr. T. Baird kindly supplied details about the patient before his admission to hospital.

\section{REFERENCES}

Meacham, G. C., et al. (1951). Blood, 6, 706

Blish Medical Jourmat, 2,897

and Barrowcliff, D. F. (1951). J. Path. Bact., 63, 552.

At the annual general meeting of the Medical Sickness Finance Corporation Ltd., held on April 27, it was reported that, during $1953,1,153$ agreements were entered into for a total of $£ 417,777$. This was an increase on the previous year's business, and the corporation continues to expand. Although the largest demand was for motor-cars, agreements were also completed for caravans, motor cycles, electrocardiographs, $x$-ray apparatus, surgery furniture, and other medical equipment. The Corporation is a subsidiary of Medical Sickness Annuity and Life Assurance Society Limited, and Sir CeCIL WAReley, P.R.C.S., is chairman.

\section{MALIGNANT DISEASE ASSOCIATED WITH VASCULAR PHENOMENA}

\author{
A. ALUN WILLIAMS, M.D., M.R.C.P.Ed. \\ Physician, Tees-side Hospitals
}

Trousseau (1877) drew attention to recurring phlebitis as the first sign of obscure visceral carcinoma. He pointed out that it may be diagnostic in certain cases, and that it may occur with cancer affecting any internal organ. To the present date 60 cases of thrombophlebitis migrans associated with carcinoma have been reported.

Wright (1952) mentions that he has seen 20 such cases, but does not report them in detail. He observes that the diagnosis of malignancy must be suspected $(a)$ whenever thrombophlebitis persists, or is migratory, in spite of adequate anticoagulant therapy in adults over 30, and with increasing suspicion in the older age groups; and (b) when there is unexplained bleeding with prothrombin times in the therapeutic (not excessive) range. Gross et al. (1951) reviewed the literature of reported cases up to 1951. Of these cases, 10 were associated with carcinoma of the stomach, 32 with carcinoma of the pancreas (head, body, and tail), 9 with lung cancer, 2 with gall-bladder cancer, 1 with carcinoma of pancreas or stomach, and 2 were undetermined. A further four cases associated with carcinoma of the lung have been reported by Fisher et al. (1951).

Raynaud's phenomenon associated with malignant disease is rare, and only three cases are reported in the literature (Pasteur and Price Jones, 1901 ; Hamilton, 1920 ; Bennett and Poulton, 1928).

The object of this paper is to report four cases of malignant disease presenting as thrombophlebitis migrans and one case presenting with Raynaud's phenomenon, and to discuss the possible relationship and pathogenesis.

\section{Case 1}

A man aged 35 was admitted to hospital on September 2, 1951 , with a history of vague malaise for seven months. Five weeks previously he had developed a thrombosis of the superficial veins of the left arm. Twelve days before admission thrombophlebitis in the left leg developed, associated with haemoptysis five days later. During the course of his illness until his death further episodes of haemoptysis occurred, the radiological appearances being compatible with pulmonary infarcts. At various times thrombophlebitis occurred in the left leg, right leg, superficial abdominal veins, dorsum of the right hand, and left external jugular vein.

Three months after admission a right-sided pleural effusion developed. Malignant cells were identified in the pleural fluid, indicative of a bronchogenic carcinoma. The liver became enlarged and nodular, and he died on January 2, 1952.

Anticoagulant therapy with ethyl biscoumacetate (" tromexan," 4-hydroxycoumarin) was maintained during his whole illness until December 22 . The maintenance dose varied between 300 and $450 \mathrm{mg}$. daily, the prothrombin concentration being kept at 10 to $25 \%$, with occasional escapes. In spite of this, thrombotic episodes occurred even with a prothrombin concentration of $10 \%$.

\section{Case 2}

This patient, a man aged 46, was admitted to hospital on March 8, 1952. One month previously thrombophlebitis 